\title{
Effect of a spacer on pulmonary aerosol deposition from a jet nebuliser during mechanical ventilation
}

\author{
C J Harvey, M J O'Doherty, C J Page, S H L Thomas, T O Nunan, D F Treacher
}

\begin{abstract}
Background - Several factors have been identified which improve nebulised aerosol delivery in vitro. One of these is the addition of a spacer to the ventilator circuit which improves aerosol delivery from a jet nebuliser to a model lung by approximately $30 \%$. The current study was designed to demonstrate whether similar improvements could be demonstrated in vivo.

Methods - Ten patients (seven men) were studied during mechanical ventilation (Siemens Servo 900C) after open heart surgery. Aerosol was delivered using a Siemens Servo 945 nebuliser system (high setting) driving a System 22 Acorn jet nebuliser (Medic-Aid) containing $3 \mathrm{ml}$ technetium-99m labelled human serum albumin $\left({ }^{99 m}\right.$ Tc-HSA $(50 \mu g)$; activity in the first nebulisation, $90 \mathrm{MBq}$; in the second nebulisation, $185 \mathrm{MBq}$ ). Central and peripheral lung aerosol deposition and the time to complete deposition were measured using a gamma camera and compared when the nebuliser was connected to the inspiratory limb using a simple $\mathrm{T}$ piece or a $600 \mathrm{ml}$ spacer.

Results - The addition of the spacer increased total lung deposition (mean (SD) percentage initial nebuliser activity) from $2.2(0.7) \%$ to $3(0.8) \%$. There was no difference in the time required to complete nebulisation $(18.2 \mathrm{~min} v 18.3 \mathrm{~min}$ respectively for $T$-piece and spacer) or in the retention of activity in the nebuliser (46.2\% $v 47 \cdot 1 \%$ respectively).

Conclusions - The combination of a spacer with a jet nebuliser increased lung deposition by $36 \%$ in mechanically ventilated patients and is a simple way of increasing drug deposition or reducing the amount of an expensive drug required for nebulisation.

(Thorax 1995;50:50-53)
\end{abstract}

Keywords: ventilator, aerosol, nebuliser.

Pharmacology,

University of

Newcastle,

Newcastle upon Tyne

NE1 7RU, UK

S H L Thomas

Reprint requests to: Dr M J O'Doherty.

Received 27 May 1994 Returned to authors 1 September 1994 Revised version received 23 September 1994 Accepted for publication 26 September 1994

The administration of nebulised drug aerosols to mechanically ventilated patients is increasing in intensive therapy units. Several studies have shown in vivo pulmonary aerosol deposition to be poor, in the range $1 \cdot 2-2 \cdot 9 \% .^{1-3}$ In vitro methods investigating pulmonary deposition, using ventilator circuits and artificial lungs, have shown a number of ways in which aerosol delivery may be improved during mechanical ventilation $^{4-6}$ including changing the type of nebuliser or ventilator settings or increasing the volume of fill of the nebuliser.

The addition of a chamber to conserve aerosol formed by jet nebulisers during the expiratory phase of respiration for subsequent delivery during inspiration has been shown almost to double aerosol deposition in spontaneously breathing subjects. ${ }^{7}$ In vitro studies have shown that the addition of a similar chamber or spacer also improves delivery of aerosol from a jet nebuliser to a lung model by at least $25 \%$ during mechanical ventilation. ${ }^{56}$ This effect has not been shown in vivo. This study was therefore designed to determine whether the addition of a spacer to a jet nebuliser improves pulmonary deposition in mechanically ventilated patients.

\section{Methods}

PATIENT SELECTION

Ten patients (seven men, eight ex-smokers and two never smokers) were studied during mechanical ventilation after open heart surgery. The study was approved by the ethics committee of West Lambeth Health District. Informed consent was obtained from all patients prior to elective open heart surgery. Measurements of forced expiratory volume in one second $\left(\mathrm{FEV}_{1}\right)$, forced vital capacity (FVC), and peak expiratory flow rate (PEFR)) were made preoperatively (table 1). Patients with pre-existing respiratory symptoms or disease were excluded as the aim of the study was to document aerosol deposition in patients with normal lungs. Studies were only performed on patients who required mechanical ventilation on clinical grounds and were commenced within a few hours of the operative procedure.

STUDY DESIGN

All patients were studied during mechanical ventilation via an endotracheal tube (Siemens

Table 1 Morphometric data, lung function, and ventilator settings in 10 ventilated patients

\begin{tabular}{lcl}
\hline & Mean & Range \\
\hline Preoperative assessment: & \multicolumn{1}{l}{} \\
Age (years) & $65 \cdot 1$ & $55-74$ \\
FEV (1) & $2 \cdot 1$ & $1 \cdot 3-3 \cdot 8$ \\
FVC (1) & $2 \cdot 6$ & $1 \cdot 6-4 \cdot 8$ \\
PEFR (1/min) & 444 & $327-657$ \\
Mechanical ventilation settings: & \\
Respiratory rate (breaths/ & 15 & $14-16$ \\
$\quad$ min) & $9 \cdot 6$ & $7 \cdot 7-11 \cdot 0$ \\
Minute volume (1/min) & 96 & $25-33$ \\
Inspiratory time (\%) & 26 & $10-20$ \\
Pause time (\%) & 11 &
\end{tabular}




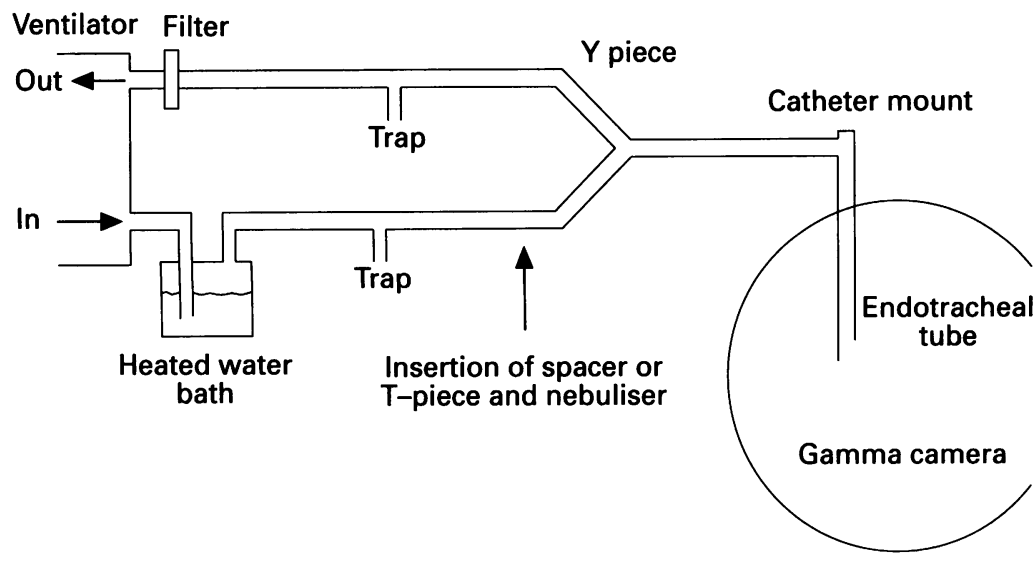

Figure 1 The circuit used to ventilate patients with a Siemens $900 \mathrm{C}$ ventilator and the site of the nebuliser, T-piece or spacer.

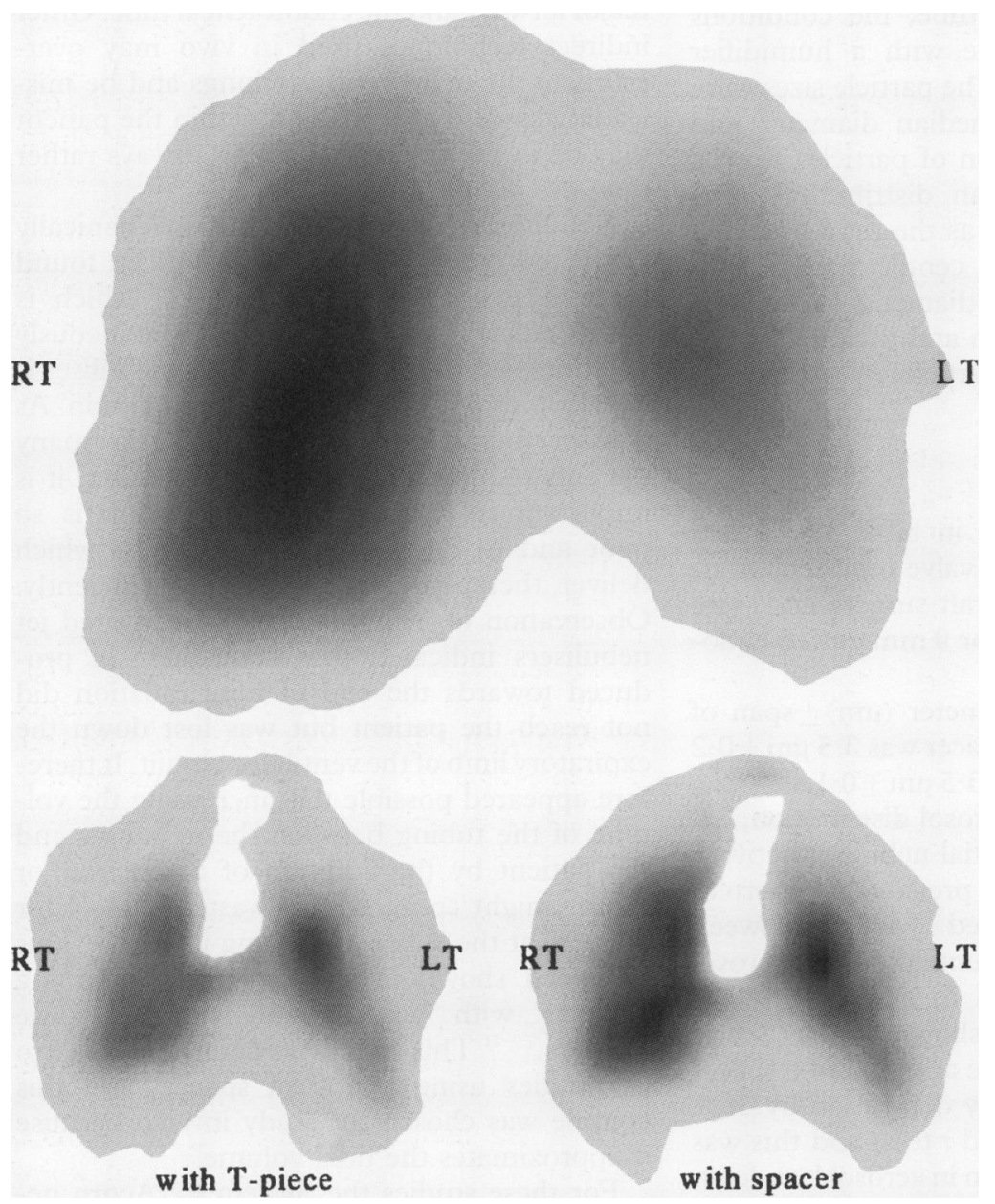

Figure 2 (A) Xenon-133 ventilation scan and (B) technetium-99m aerosol deposition scan in the same patient with and without the spacer. The two images of aerosol deposition are scaled to the maximum deposition in the spacer deposition. In the deposition images the trachea has been masked.

Servo $900 \mathrm{C}$ ventilator in volume mode) using the ventilator circuit shown (fig 1). Before each study the ventilator settings were adjusted to optimise the patient's gas exchange and were thereafter kept constant (table 1). A portable gamma camera (IGE Portacamera, (SFOV), IGE Medical Systems, Berkshire, UK) fitted with a diverging collimator was positioned anteriorly. The tissue attenuation through the thorax was measured using a $47 \mathrm{~cm}$ diameter cobalt-57 flood source after a previously described method. ${ }^{38}$ Pulmonary deposition was measured using the gamma camera as pre- viously described ${ }^{38} \mathrm{~A}$ xenon-133 scan was performed before nebulisation to define lung edges and regions. This was obtained by injection of $110 \mathrm{MBq}$ of gas through the proximal end of the endotracheal tube immediately before the inspiratory phase of the ventilator. A breath hold image was obtained by suspending ventilation for 10 seconds at the end of the inspiratory cycle whilst an anterior image was acquired (fig $2 \mathrm{~A}$ ). The expired ${ }^{133} \mathrm{Xe}$ was collected via the expiratory port of the ventilator into a Douglas bag until washout was complete.

A Siemens Servo 945 nebuliser system (high setting) was used to administer a solution of technetium-99m labelled human serum albumin $\left({ }^{99 \mathrm{~m}} \mathrm{Tc}-\mathrm{HSA} 50 \mu \mathrm{g}\right)$ in $3 \mathrm{ml}$ saline using a System 22 Acorn jet nebuliser (Medic-Aid, Sussex, UK). In the first study the nebuliser was positioned via a $\mathrm{T}$-piece in the inspiratory limb set $12 \mathrm{~cm}$ from the Y piece. In the second study a $600 \mathrm{ml}$ spacer was included in the delivery system at the same distance (fig 1). The order of these two studies was randomised. Thus, two studies of aerosol deposition were performed in each patient, with activities in the nebuliser of $90 \mathrm{MBq}$ in study 1 and $185 \mathrm{MBq}$ in study 2. Nebulisers were placed on the camera face before and after nebulisation. Study 2 was performed immediately after completion of study 1 using the same ventilator settings, experimental methods, and nebulisation equipment. Since the administration of aerosols via a jet nebuliser during mechanical ventilation requires inspiratory phase actuation, the volume of gas necessary to drive the nebuliser has to be taken into account when setting the tidal volume. The respiratory minute volume was therefore adjusted manually on the ventilator to compensate for the consequent rise in expired minute volume when the nebuliser is in use.

During nebulisation pulmonary deposition of ${ }^{99 \mathrm{~m}}$ Tc-HSA was measured continuously via the gamma camera (anterior projection) in 15 second frames. Following completion of nebulisation, anterior and posterior static scans of the lungs were taken, each over 300 second periods. The gamma camera was also used to measure activity in the T-piece, exhalation filter, and spacer after nebulisation. The deposition in each lung, tracheal region, and extrapulmonary apparatus was determined by computer analysis. Regional lung aerosol deposition was subdivided into central and peripheral distribution. The lung edges were defined by the $20 \%$ isocount contour of the ${ }^{133} \mathrm{Xe}$ image. The lung was divided into thirds by height and then the central region was defined as an area drawn in the middle third from the medial edge extending midway across the lung. ${ }^{3}$ The rest of the lung was regarded as the peripheral region. The deposition in the lung from study 2 was corrected for deposition from study 1 by simple subtraction after correction for decay and background activity.

\section{PARTICLE SIZING}

The aerosol particle size was determined in a separate experiment using a Malvern laser 
Table 2 Mean (SD) aerosol deposition in 10 ventilated patients with and without a spacer

\begin{tabular}{|c|c|c|}
\hline & T-piece & Spacer \\
\hline $\begin{array}{l}\text { Pulmonary deposition: } \\
\text { Both lungs } \\
\text { Peripheral } \\
\text { Central } \\
\text { Right lung } \\
\text { Left lung }\end{array}$ & $\begin{array}{l}2 \cdot 19(0 \cdot 73) \\
2 \cdot 00(0 \cdot 69) \\
0 \cdot 19(0 \cdot 1) \\
1.47(0.58) \\
0 \cdot 71(0 \cdot 25)\end{array}$ & $\begin{array}{l}2.99(0.78)^{*} \\
2.54(0.70)^{*} \\
0.45(0.2)^{*} \\
2.04(0.66)^{*} \\
0.95(0.28)^{*}\end{array}$ \\
\hline $\begin{array}{l}\text { Extrapulmonary deposition: } \\
\text { Tracheal endotracheal tube } \\
\text { Exhalation filter } \\
\text { T-piece or spacer } \\
\text { Nebuliser retention }\end{array}$ & $\begin{array}{r}1 \cdot 14(0 \cdot 49) \\
18 \cdot 10(2 \cdot 48) \\
1.09(0 \cdot 39) \\
46 \cdot 20(7 \cdot 3)\end{array}$ & $\begin{array}{r}1.48(0.93) \\
15.45(1.5) \\
5.65(1.5) \\
47 \cdot 10(7.9)\end{array}$ \\
\hline $\begin{array}{l}\text { Time to completion of } \\
\text { nebulisation (min) }\end{array}$ & $18 \cdot 2(5)$ & $18 \cdot 3(4 \cdot 5)$ \\
\hline
\end{tabular}

${ }^{*} \mathrm{p}<0.02$, two tailed paired $t$ test.

mastersizer. The aerosol was measured at the tip of the endotracheal tube; the conditions were otherwise the same with a humidifier operating in the circuit. The particle sizes were recorded as the mass median diameter and the span. The distribution of particles from a nebuliser have a gaussian distribution. The span is therefore defined as the ratio obtained by subtracting the 10 th centile particle size diameter from the larger diameter of the 90th centile particle dimension and dividing by the size of the 50th centile particle.

\section{Results}

Patient details are given in table 1. All had undergone either cardiac valve replacement or coronary artery bypass graft surgery and were intubated with an $8 \mathrm{~mm}$ or $9 \mathrm{~mm}$ cuffed endotracheal tube.

The mass median diameter $(\mu \mathrm{m}) \pm$ span of the aerosol without the spacer was $3.5 \mu \mathrm{m} \pm 0.2$ and with the spacer was $3.5 \mu \mathrm{m} \pm 0 \cdot 1$.

The pattern of radioaerosol distribution, expressed as percentage initial nebuliser activity, is given in table 2. As previously reported, aerosol deposition varied widely between patients. In the left lung ventilation and aerosol deposition were reduced in all patients; the scan of one patient is shown in fig 2 and clearly illustrates this. The addition of a spacer increased total pulmonary deposition by $36 \%$ $(\mathrm{p}<0.02$, paired two tailed $t$ test) and this was associated with a reduction in aerosol loss down the expiratory limb of the circuit. Approximately $30 \%$ of the initial nebuliser activity is unaccounted for and is presumed to be deposited in the ventilator circuit. It was not possible to measure this because of technical difficulties in scanning long lengths of tubing, traps, and a heated water bath.

\section{Discussion}

Improving the efficiency of aerosol delivery by nebulisers is worthwhile because this may increase their clinical value and reduce the expense of drugs administered using this route. There are a number of factors that affect aerosol delivery from a nebuliser during mechanical ventilation which have been identified in vitro. ${ }^{4-6}$ Delivery may be increased by optim- ising the nebuliser position, changing the gas flow to the nebuliser, increasing the inspiratory fraction, reducing the respiratory rate or minute volume, increasing nebuliser fill, or reducing humidity. ${ }^{4}$ These in vitro measurements indicate possible ways of increasing delivery to patients but they cannot allow for the specifics of airway geometry and humidity, lung disease, or mucus secretion. Neither can they predict the site of deposition within the bronchial tree. Thus, in vivo studies are needed to quantify the amount deposited in the trachea, major airways, and "peripheral" lung. In vitro aerosol delivery will be greater than the in vivo lung deposition since the filter used in the model ventilator circuit captures all delivered aerosol particles and cannot quantify the proposition that would be exhaled or deposited in the major airways and the endotracheal tube. Other indirect techniques used in vivo may overestimate the delivery to the lungs and be misleading since the deposition within the patient may be in the trachea or major airways rather than the peripheral lung. ${ }^{8}$

Nebulised aerosol deposition in mechanically ventilated patients has previously been found to be between $1 \cdot 2 \%$ and $2 \cdot 9 \%,,^{1-3}$ which is not as efficient as deposition in spontaneously breathing subjects even though the nebuliser is usually only activated during lung inflation. As aerosol therapy may be of value in many patients requiring mechanical ventilation, it is important to establish why deposition is so poor and to design nebuliser systems which deliver therapeutic aerosols more efficiently. Observation of inspiratory phase activated jet nebulisers indicated that some aerosol produced towards the end of lung inflation did not reach the patient but was lost down the expiratory limb of the ventilator circuit. It therefore appeared possible that increasing the volume of the tubing between the nebuliser and the patient by the addition of a reservoir or spacer might conserve this wasted aerosol for delivery at the subsequent lung inflation. This has been shown in spontaneously breathing subjects with aerosol and metered dose inhalers. $^{79-11}$ This effect was confirmed in vitro by studies using a $600 \mathrm{ml}$ spacer $^{5}$ and this volume was chosen for study in vivo because it approximates the tidal volume.

For these studies the System 22 Acorn nebuliser and Siemens 945 nebuliser activating system were chosen because they are commonly used on intensive care units and produce suitably sized particles, small enough to pass through the ventilator circuit and endotracheal tubing to reach the patient. The experimental protocol, involving two separate but sequential experiments in the same patient, was chosen because it is convenient and allows a paired comparison. Its validity and reproducibility have been reported previously. ${ }^{3}$

The results confirmed that total pulmonary deposition is consistent with values found in previous studies as is the percentage deposited in the endotracheal tube/trachea and retained in the nebuliser. ${ }^{3}$ Pulmonary deposition and ventilation were reduced in the left lung. This was most probably due to the opening of the 
pleura on the left side during the operative procedure. This would result in the greater degree of collapse of the left lung with the lower lobe expansion being most affected postoperatively. The results also showed that the improvement in aerosol delivery with a spacer obtained in vitro could be produced in vivo, and that aerosol delivery was significantly increased to all regions of the lung. This is an important observation as it illustrates the value of in vitro studies using lung models for predicting the effects of nebuliser or ventilator circuit modifications on aerosol delivery in vivo. The increase was associated with a reduction in aerosol entering the expiratory limb of the circuit and this is consistent with the way the spacer works. The observed improvement is modest and is unlikely to be of great clinical importance when used alone. However, when used in combination with other modifications - for example, increased volume of fluid in the nebuliser - it may make a useful contribution to an overall improvement in aerosol deposition. It was to be expected that the improvement in delivery with an aerosol reservoir would not be as great during volume cycled ventilation as in spontaneously breathing subjects, as in the former the nebuliser is inspiratory phase activated and a larger proportion of the aerosol is produced during lung inflation. Larger improvements would be predicted if the nebuliser was driven throughout the respiratory cycle, as happens with pressure cycled ventilation. It may also be that
$600 \mathrm{ml}$ is not the optimum spacer volume and further studies in this area would be useful.

The authors thank the nursing staff and technicians of Mead Ward, St Thomas' Hospital, especially Valerie Arnold, for their cooperation and technical assistance. We are particularly grateful to Mr C Young, Mr G Venn, Dr C Apps, Dr T Hunt, and Dr $\mathrm{R}$ Linton for allowing them to study their patients.

1 Fuller HD, Dolovich MB, Posmituck G, Wong Pack W, Newhouse MT. Pressurised aerosol versus jet aerosol delivery to mechanically ventilated patients. Comparison of
dose to the lungs. Am Rev Respir Dis 1990;141:440-4.

2 MacIntyre NR, Silver RM, Miller CW, Schuler F, Coleman RE. Aerosol delivery in intubated mechanically ventilated patients. Crit Care Med 1985;13:81-4.

3 Thomas SHL, O'Doherty MJ, Fidler HM, Page CJ, Treacher DF, Nunan TO. Pulmonary deposition of a nebulised aerosol during mechanical ventilation. Thorax 1993;48:154-9.

4 O'Riordan TG, Greco MJ, Perry RJ, Smaldone GC. Nebulizer function during mechanical ventilation. $\mathrm{Am}$ Rev bulizer function during mech

5 O'Doherty MJ, Thomas SHL, Page CJ, Treacher DF, Nunan TO. Delivery of a nebulised aerosol to a lung model during mechanical ventilation: effect of ventilator settings and nebuliser type, position and volume of fill. Am Rev Respir Dis 1992;146:383-8.

6 Thomas SHL, O'Doherty MJ, Page CJ, Treacher DF, Nunan TO. Delivery of ultrasonic nebulised aerosols to a lung model during mechanical ventilation. Am Rev Respir Dis 1993;148:872-7.

7 Thomas SHL, Langford JA, George RDG, Geddes DM. Improving the efficiency of drug administration with jet nebulisers. Lancet 1988;i:126.

8 O'Riordan TG, Palmer LB, Smaldone GC. Aerosol deposition in mechanically ventilated patients: optimising nebuliser delivery. Am ₹ Respir Crit Care Med 1994;149: 214-9.

9 Newman SP, Moren F, Pavia D, Little F, Clarke SW. Deposition of pressurised suspension aerosols inhaled through extension devices. Am Rev Respir Dis 1981;124: through

10 Newman SP, Millar AB, Lennard-Jones TR, Moren F, Clarke SW. Improvement of pressurised aerosol deposition with Nebuhaler spacer device. Thorax 1984;39:935-41.

11 Vidgren MT, Paronen TP, Karkkainen A, Karjalainen P. Effect of extension devices on drug deposition from inhalation aerosols. Int $\mathcal{F}$ Pharm 1987;39:107-12. 